\title{
Rate of Inappropriate Imaging Utilization by the Emergency Department in Community Hospitals
}

\author{
Sébastien Robert ${ }^{1 *}$, Murray Asch ${ }^{2}$, Larry Nijmeh ${ }^{2}$ \\ ${ }^{1}$ Faculty of Medicine, University of Ottawa, Ottawa, Canada \\ ${ }^{2}$ Lakeridge Health, 1 Hospital Court, Oshawa, Canada \\ Email: *srobe017@uottawa.ca
}

How to cite this paper: Robert, S., Asch, M. and Nijmeh, L. (2018) Rate of Inappropriate Imaging Utilization by the Emergency Department in Community Hospitals. Open Journal of Radiology, 8, 281-292. https://doi.org/10.4236/ojrad.2018.84031

Received: October 29, 2018

Accepted: December 2, 2018

Published: December 5, 2018

Copyright (c) 2018 by authors and Scientific Research Publishing Inc. This work is licensed under the Creative Commons Attribution International License (CC BY 4.0).

http://creativecommons.org/licenses/by/4.0/

\section{(c) (i) Open Access}

\begin{abstract}
Objective: To retrospectively analyse the use of imaging studies in the Emergency Department of community hospitals using evidence based guidelines and clinical judgement. Methods: Medical records of 661 patients who visited the Emergency Department (ED) in 2015 and underwent imaging studies were reviewed. The Canadian Association of Radiologists, American College of Radiologists and Choosing Wisely Canada guidelines were used to determine the appropriateness of imaging studies. The use of prior patient imaging, the rate at which studies were repeated and the respective impacts on patient management of the imaging studies were also examined. Results: Of the 1056 imaging studies reviewed, 228 (22\%) were found to be clinical situations where no imaging study was indicated while $168(16 \%)$ were considered a suboptimal choice of imaging study or modality. When no study was recommended, a positive impact on the diagnosis was noted in 105 (46\%) cases and on patient management 83 (36\%) times. Notably, 219 (21\%) patients had a relevant examination performed in the last 30 days, and 147 (14\%) reports noted that the results of the prior study also concurred with the imaging study evaluated. Conclusion: In this study, 228 (22\%) radiographs and CT studies, excluding MVC related imaging and extremity imaging, were not indicated based on appropriateness criteria and consequently had a limited impact on patient management. This supports the need for increased clinical decision support for ED physicians, regional health information exchanges and consideration of Computerized Physician Order Entry in the ED with embedded appropriateness criteria at the point of ordering.
\end{abstract}

\section{Keywords}

Diagnostic Imaging, Evidence-Based Guidelines, Emergency Department, 
Community Hospital

\section{Introduction}

As overall use and availability of various medical imaging modalities continues to climb, the impact of imaging costs on health care systems has gained increased attention. In recent years, appropriateness of diagnostic imaging has been a significant topic of discussion in the medical community [1]. An imaging examination can be labelled as inappropriate for a variety of reasons, including the unnecessary repetition of a satisfactory imaging study and the inability of the investigation to answer the clinical question and consequently, contribute positively to patient management [2]. Since all imaging modalities come with a cost, it is important that patients receive only appropriate imaging which will aid and contribute to their management [2]. Estimates of the rate of inappropriate imaging have largely varied in literature, making it apparent that no clear consensus has been reached [3]. As patient safety continues to be a developing point of emphasis, decreasing general radiation exposure, unnecessary patient anxiety and the need for various consequent invasive procedures are important and desirable goals [2].

Various research articles have been able to demonstrate the positive impact of using available guidelines to help decrease imaging use [4]. In Ottawa, the establishment of four decision rules: the Ottawa Ankle Rule, the Ottawa Knee Rule, the Canadian C-Spine Rule and the Canadian CT Head Rule resulted in a 20\% $30 \%$ overall decrease in imaging [5]. However, in a recent survey of emergency physicians, it was found that although nearly every Canadian physician was aware of the Canadian Cervical Spine Rule, only 73\% made use of it in relevant situations [6]. These statistics help to enlighten the issue of variable application of evidence-based recommendations.

The impact of an inappropriate test request can extend much further than just initial imaging [7]. A recent Canadian study found that $26.1 \%$ of all CT and MRI radiology reports recommended the completion of further follow-up imaging studies [8]. It is clear that the snowball effect, which often takes place with diagnostic tests, can be another potential area of improvement in resource management.

Research has shown that a lack of information transfer between institutions tends to be a significant factor in repeat imaging studies [9]. A few studies have concretely evaluated the benefits of a Health Information Exchange (HIE) system which allows providers to easily share clinical information between centers [10]. In a Boston study, a successful transfer of prior imaging was shown to lead to a $17 \%$ reduction in all subsequent imaging performed by the Emergency Department to which the patient was transferred [11].

Since little data is available regarding the appropriateness of imaging in 
community hospitals, development of strategies to measure and support appropriate use should be of priority. This research project aims to provide insight through the evaluation of the application of imaging guidelines in Canadian community Emergency Departments. Through this analysis, we aimed to acquire retrospective data of imaging guideline application while simultaneously offering opportunities for practice improvements through the identification of potential areas of improvement.

\section{Methods}

This study was approved by the local Hospital Institutional Review Board. A retrospective review of Emergency Department (ED) patients who underwent imaging studies during their admission was undertaken. The first week of February of 2015 was randomly selected (utilizing http://www.random.org/) to act as our main patient database.

Initial patient inclusion criteria included all patients who were admitted during the selected week to one of the three Emergency Departments part of the health partnership under study. Distinction between the different sites was not made in the analysis of the study. These community hospitals receive over 200,000 Emergency department visits yearly and constitute one of the largest community hospital systems in the province of Ontario.

Focus was placed on radiation emitting modalities which expose patients to potential long-term health related consequences, thus adding a supplementary element of importance to the inappropriate vs. appropriate imaging conversation. Patients were included if they had undergone either a radiograph or a CT scan during the selected week. Furthermore, if the same patients returned to the Emergency Department within a four-month period and required further radiograph or CT imaging, these cases were also retrospectively evaluated. This was done to collect data on trends of patients returning to the Emergency Department with either the same or a new clinical presentation. Finally, an evaluation of the usage of prior patient imaging, the rate at which studies were repeated and their respective impacts on patient management was also completed.

Exclusion criteria included imaging studies performed for motor vehicle collision (MVC) related traumas. These were excluded as patients involved in MVC usually elicit many simultaneous studies and often include certain legal repercussions given their higher risk nature. We also excluded all upper and lower extremity imaging, from the clavicle to the fingers and from the femur to the toes. The rational for the exclusion was two-fold. First, many extremity injuries have specific imaging rules that physicians can follow such as the Ottawa Ankle Rule. Thus, the debate of appropriate vs. inappropriate imaging is far less prevalent in the medical community for these types of imaging studies. Furthermore, upper and lower extremity imaging were excluded from analysis to help decrease the overall number of cases to be reviewed in this study, so that focus could be placed on the areas of greater concern. All other areas including the head, chest, 
abdomen, pelvis and hips were included and consequently focused on in the analysis.

The data collected and analysed consisted of patient clinical history, the date of the ED visit, recent relevant imaging information, the imaging study performed as well as the clinical information included in its request, Radiologist report summary and final ED physician and Radiologist diagnoses. The review process started with the examination of the scanned emergency worksheet, for a given patient, which established the patient's symptoms and general presentation. The emergency physician's main clinical question was confirmed with the use of the imaging request documentation. Following this, a review of the patient's prior imaging was completed to establish whether or not relevant priors were available. If this was the case, this was further investigated to determine if the available prior imaging was for a similar presentation and consequently, if it could have been used to answer the clinical question. Then, the radiologist's report was read, followed by a second review of the emergency documentation to see if the radiologist recommendations or suggested diagnosis affected patient management. Finally, comparing the patient presentation as well as the clinical question to the guidelines made it possible to establish whether or not the study was indicated. Only patient information made available through the hospital clinical information system was considered.

\section{Establishing the Support of a Study}

Reference was made to the Diagnostic Imaging (DI) guidelines from the Canadian Association of Radiologists (CAR), the American College of Radiologist (ACR) as well as Choosing Wisely Canada [12] [13] [14]. Patient cases were classified as appropriate if the imaging requested followed at least one of these guidelines. A study could also be classified as suboptimal if the guidelines recommended the use of either a different view, for example a standard chest radiograph vs. a rib view, or the use of a different modality, such as an abdominal ultrasound instead of an abdominal radiograph, based on the clinical picture. The initial classification was completed by reviewing emergency physician documentation, considering recent priors and identifying the main diagnosis to rule out. Then, evaluating the final diagnosis, patient treatment following the completion of the study and whether or not recommendations included in the radiologist reports were followed, allowed for the determination of overall impact on diagnosis and management. In cases where patient presentation was significantly complex, when there was inadequate clinical information, when imaging had to be repeated due to poor image quality or when no clear commendations were found in the set of guidelines referenced, the support of the study by guidelines was deemed indeterminate.

For instance, an individual presenting with fever and a productive green cough who undergoes a standard PA chest radiograph to rule out pneumonia is a study which is supported by both ACR and CAR guidelines and thus, was 
deemed as appropriate. However, if this same individual returned to the emergency department a few days later with the same presentation, the repeat PA chest radiograph performed would be classified as no study indicated, as it is not a practice supported by guidelines and would likely not provide any supplementary information. Finally, an individual who suffers a fall, has a decreased level of consciousness and cervical tenderness constitutes an indication for a CT scan instead of a cervical radiograph, thus making this kind of study fall under the wrong imaging modality category. The analysis was completed in this fashion to illustrate the potential impact of implementing well recognized and readily accessible guidelines in the decision-making process.

\section{Results}

In total, $661 \mathrm{ED}$ patients fell within the established criteria and their cases were analysed. The patient demographic consisted of 304 males and 357 females. In total, 1056 imaging studies and their respective cases were analysed in this study. Patients in this study underwent a subsequent imaging study for their presenting illness 325 (31\%) times. Overall, 332 (32\%) studies analysed resulted in positive findings as reported by the radiologist in contrast to $721(68 \%)$ imaging studies showing either negative or indeterminate findings. For example, if an emergency physician wanted to rule out the possibility of pneumonia, an area of lung consolidation mentioned in the report was considered a positive finding. However, if for this same presentation a new sclerotic bone lesion was identified, this was noted as incidental.

\subsection{The use of Imaging Guidelines}

With the use of the CAR, ACR and the Choosing Wisely Canada guidelines, it was determined that in 228 (22\%) cases, no studies were recommended based on the clinical presentation. In 168 (16\%) cases, the incorrect imaging study or imaging modality was used (Table 1).

\subsection{The Impact of Imaging Guidelines on Patient Care and Management}

Studies supported by guidelines eliminated a suspected diagnosis and/or suggested a new diagnosis in over three quarters of the studies evaluated (Table 2).

Table 1. The application of general imaging guidelines to imaging decision making in the Emergency Department.

\begin{tabular}{cccc}
\hline & Radiographs & $\begin{array}{c}\text { Computed } \\
\text { Tomography }\end{array}$ & Totals \\
\hline Completed study supported by guidelines & $44 \%$ & $82 \%$ & $51 \%$ \\
No study recommended in clinical context & $26 \%$ & $4 \%$ & $22 \%$ \\
Suboptimal imaging modality or study completed & $19 \%$ & $4 \%$ & $16 \%$ \\
Indeterminate or insufficient clinical context & $11 \%$ & $10 \%$ & $11 \%$ \\
\hline
\end{tabular}


Table 2. Impact of general imaging guidelines on diagnosis, treatment and management.

\begin{tabular}{|c|c|c|c|c|}
\hline & $\begin{array}{l}\text { Completed study } \\
\text { supported by } \\
\text { guidelines }\end{array}$ & $\begin{array}{l}\text { No study } \\
\text { recommended in } \\
\text { clinical context }\end{array}$ & $\begin{array}{l}\text { Suboptimal imaging } \\
\text { modality or study } \\
\text { completed }\end{array}$ & $\begin{array}{l}\text { Indeterminate or } \\
\text { insufficient clinical } \\
\text { context }\end{array}$ \\
\hline \multicolumn{5}{|c|}{ Result obtained from imaging study } \\
\hline Positive & $36 \%$ & $36 \%$ & $11 \%$ & $32 \%$ \\
\hline Negative & $53 \%$ & $50 \%$ & $69 \%$ & $55 \%$ \\
\hline Inconclusive & $11 \%$ & $14 \%$ & $20 \%$ & $13 \%$ \\
\hline \multicolumn{5}{|c|}{ Effect of imaging study on diagnosis } \\
\hline Eliminated or suggested new diagnosis & $77 \%$ & $39 \%$ & $35 \%$ & $72 \%$ \\
\hline Supported suspected diagnosis & $12 \%$ & $7 \%$ & $4 \%$ & $9 \%$ \\
\hline Previous established diagnosis unchanged & $4 \%$ & $32 \%$ & $4 \%$ & $9 \%$ \\
\hline Unable to eliminate the main suspected diagnosis & $3 \%$ & $19 \%$ & $54 \%$ & $3 \%$ \\
\hline Positive or negative result ignored & $4 \%$ & $4 \%$ & $4 \%$ & $1 \%$ \\
\hline \multicolumn{5}{|c|}{ Effect of study on patient management } \\
\hline Eliminated need for treatment and further imaging & $32 \%$ & $15 \%$ & $13 \%$ & $22 \%$ \\
\hline Modified therapeutic approach & $35 \%$ & $9 \%$ & $7 \%$ & $23 \%$ \\
\hline Supported suspected therapeutic approach & $14 \%$ & $12 \%$ & $3 \%$ & $10 \%$ \\
\hline Further imaging evaluations needed & $12 \%$ & $5 \%$ & $21 \%$ & $15 \%$ \\
\hline Indeterminate effect & $5 \%$ & $38 \%$ & $41 \%$ & $21 \%$ \\
\hline No effect & $1 \%$ & $21 \%$ & $15 \%$ & $3 \%$ \\
\hline
\end{tabular}

These studies had a positive effect on the diagnosis 484 (89\%) times and a positive effect on patient management in 441 (81\%) presentations (Table 2). They also resulted in less inconclusive findings than all other groups (Table 2). In contrast, when no study was recommended, a positive effect on the diagnosis was found in 105 (46\%) cases. Furthermore, when a suboptimal imaging study or imaging modality was used for patient assessment, a positive effect was noted in $65(39 \%)$ cases (Table 2). A positive effect on patient management was also noted in $83(36 \%)$ and $38(23 \%)$ cases respectively for both these groups (Table 2).

When a suboptimal imaging study or modality was used according to the guidelines referenced, significantly fewer studies resulted in a positive finding resulting in more negative and indeterminate results (Table 2). These studies also required that further studies be completed for 59 (35\%) patient presentations and were unable to eliminate the main suspected diagnosis 90 (54\%) times (Table 2).

\subsection{The Use of Relevant Prior Imaging}

In our patient population, it was noted that $655(62 \%)$ cases had relevant priors available. Furthermore, 107 (10\%) of the imaging studies requested by the ED 
physicians had relevant prior imaging performed less than a week before presenting to the ED. This number increased to $219(21 \%)$ when looking at priors within 1 month of the ED visit and 408 (39\%) when considering a 6-month window. Out of the 1056 imaging study reports analysed, 147 (14\%) of these concurred with a previously performed imaging study. Of these, 18 (12\%) were the same study performed less than two days prior to patient presentation. Moreover, the same study was performed less than 1-week prior in $38(26 \%)$ cases where a concordance was noted. The greatest percentage of studies concurring with previous studies was seen in clinical situations where no studies were recommended (28\%). Furthermore, 72 (32\%) of these studies did not change the previously established diagnosis, a value far greater than all other groups (Table 2).

The prior imaging studies referred to include imaging studies performed on site, in partnership hospitals or in other outpatient clinical settings that are part of the Hospital Diagnostic Imaging Repository Services (HDIRS). Notably, it was also observed that 6 patients in this study had a foreign relevant prior which was not accessible to the ED staff, but which had been completed within a month of the patient presentation. It is to be noted that the existence of these prior foreign studies was not considered when determining the appropriateness of the studies being analysed.

\section{Discussion}

\subsection{The Impact of Imaging Guidelines}

Although recent estimates have greatly varied based on imaging types and research design, the results obtained appear to suggest that the rate at which unsupported radiograph and CT imaging are being completed in Canadian centers may in fact resemble the higher estimates found in global literature [2].

A potential solution to support appropriate imaging utilization would be the implementation of Computerized Physician Order Entry (CPOE). An enhanced CPOE system, equipped with alerts at the point of ordering which would highlight prior imaging studies from Provincial DI repositories, paired with clinical decision support around appropriate diagnostic imaging choices, could provide an important positive support to clinicians. CPOE does however come with its own set of challenges, most notably, physician acceptance of recommendations and overall software limitations [15]. CPOE also introduces noteworthy challenges in ED workflow, but could add significant value if it is well designed, optimized for providers and supports clinical decision making with minimal impact to productivity. Furthermore, advanced hospital information systems could offer the added benefit of improved ED provider-radiologist communication through provision of key clinical information which is often lost in paper documentation and non-physician order entry. As evidence based clinical decision support systems have previously shown some effectiveness in increasing the rate of appropriate imaging use, the potential practice improvement opportunities 
through its implementation in a community hospital is noteworthy [16].

Our findings strongly indorse the implementation of such a system, as unsupported studies were found to have significantly less positive impact on diagnosis and overall patient care (Table 2). When no study was indicated, the previously established diagnosis remained unchanged in nearly one third of cases (Table 2). This is a finding of note as many of the contraindicated studies were simply repeats or follow-up imaging of previously confirmed diagnoses being completed in a shorter delay than recommended by the guidelines. Implementation of CPOE, alerts and quick reference to guidelines may provide the tools necessary to significantly decrease this number.

Another simple potential point of focus for practice improvement includes placing more emphasis on the importance of good protocoling. Simple strategies such as the training of technical staff and the implementation of added elements in a checklist prior to the completion of an imaging request can ensure that radiologist have more of the necessary information to make an informed decision [16]. Nevertheless, implementation of such criteria has not always proven to be an efficient methodology, as ordering physicians can learn to modify terminology to ensure a specific study is completed, thus adding a supplementary layer of complexity [17].

It is however worth noting that unsupported and contraindicated studies did have a positive impact on patient care in a multitude of instances. Whether this unexpected high number came as a result of incomplete documentation rendering many of these studies as falsely non-indicated or as a result of physician experience and intuition is difficult to accurately determine. Nevertheless, this suggest that perhaps our overall focus should be placed on significantly decreasing instead of completely eliminating these types of studies, by allowing physicians to override a CPOE alert in clinical situations where guidelines do not fully consider certain elements of a clinical picture.

As of January 1st 2020, legislation in the United-States will be enforcing this general direction of practice under the Protecting Access to Medicare Act [18]. Physicians will be required to consult specific appropriateness imaging guidelines for radiology to receive reimbursement through Medicare [19]. By implementing this strategy and adding financial implications to the following of imaging guidelines, the goal is to reduce unsupported and clinically unjustified imaging studies. If through a system-based approach even a small percentage of the observed unwarranted imaging studies were eliminated, there is potential of great financial savings, decreased radiation exposure to patients and elimination of many follow-up studies which are performed due to incidental findings.

\subsection{Repeat Studies and the Insufficient Use of Prior Imaging}

Research has suggested that the issue of unnecessarily repeated diagnostic imaging studies is still an area which could use great improvement [19]. A recent Ontario study demonstrated through a large-scale analysis that cross-sectional imaging of the head, chest, abdomen and pelvis were reimaged with the use of 
the same modality at the rate of $12.8 \%$ within a 90 -day period [19]. Furthermore, they showed that this frequency of repeats decreased in areas where a shared online imaging repository was used [19]. Similarly, our findings demonstrated that $231(22 \%)$ of the studies evaluated had a relevant prior image performed within a 180-day period.

Implementation of a Health Information Exchange database with integrated alerting at the time of DI ordering could potentially help address this issue [20]. Efficient access to all patient prior imaging holds great quality of care impact and recent estimates have demonstrated overall positive savings associated with such access [21]. Algorithms which would flag studies done within a certain time interval or anatomical proximity to the one being requested would further support more informed and efficient diagnostic imaging approaches by helping to decrease, most notably, the number of unsupported studies [20].

Although the hospital system analysed in this study does belongs to a shared imaging repository extending to many other hospitals and clinics, this is not all inclusive. As was the case for 6 individuals analysed in this review, previous foreign imaging studies may remain inaccessible with this type of system. Furthermore, the repository is not integrated with the hospital information system, thus making the process of looking up previous imaging studies a multi-step process for providers. In the situations observed, the unavailable priors had been performed less than 1 month before the patients presented to the Emergency Department suggesting that some could have potentially negated the need for new imaging. We argue that moving to a single shared imaging repository for the Province which includes a system of notifications that could alert providers of the availability of prior imaging studies at the point of ordering could further decrease the observed incidence of repeat imaging.

\section{Limitations}

This study relied upon the review of many written paper-based ED records which poses legibility and completeness challenges. It is therefore likely that certain studies may have in fact been supported by guidelines, but the available physician documentation was not complete. Furthermore, occasional clerical errors in assigning the correct ordering physician were noted which presents a challenge in studying physician utilization in a non-CPOE environment. Studies may have been ordered under the name of the emergency physician, even if the patient had been transferred to a new most responsible provider who was the actual ordering physician, thus contributing to a small overestimation in the number of studies requested by the emergency department. Lastly, transcription errors, although not directly evaluated, have been described previously in literature as a noteworthy source of error in imaging request and thus constitute another area of potential limitation [22].

\section{Conclusions}

In summary, 228 (22\%) radiographs and CT studies requested by the Emergency 
Department in a community hospital, excluding MVC related imaging and extremity imaging, did not meet any of the CAR, ACR or Choosing Wisely Canada guidelines. Additionally, in 168 (16\%) cases, a suboptimal imaging study or imaging modality was utilized. In all cases of unsupported imaging use, a positive impact on patient care and management was noted less than $50 \%$ of the time. Most patients had relevant prior imaging, with 107 (10\%) patients having one within one week prior to their ED presentation.

Significant opportunities exist regarding improving appropriate utilization of diagnostic imaging resources in the ED. Although clearly multifactorial, we suggest that there is potential for major system benefit especially with physician engagement. Focus should be placed on developing optimized CPOE with embedded alerting and clinical decision support that fits provider workflow, maximises efficiency and minimises patient harm. Furthermore, although previous imaging studies may be automatically retrieved and made available in certain systems or departments at the time of imaging review, it is currently not available at the time of ordering. By implementing a Health Information Exchange system paired with an alert system, the amount of imaging studies which are repeated due to inaccessibility or lack of provider awareness could be decreased.

Building towards a future where prior imaging is consistently made available to physicians at the point of care, with appropriate use guidelines being integrated in the decision-making process may lead to significant reductions in unnecessary imaging. Continued physician and patient education around appropriate use of imaging is also key. Considering the growing use of diagnostic imaging, such strategies could play an important role in improving the quality and safety of patient care, reducing ED wait times and overall health system costs. Supporting appropriate utilization at the point of care with embedded and up to date guidelines holds great potential in improving resource management which extends far beyond the completion of a single inappropriate test.

\section{Conflicts of Interest}

The authors declare no conflicts of interest regarding the publication of this paper.

\section{References}

[1] Morrison, A. (2013) Appropriate Utilization of Advanced Diagnostic Imaging Procedures: CT, MRI, and PET/CT. Environmental Scans, 39, 1-25.

[2] Fraser, J. and Reed, M. (2013) Appropriateness of Imaging in Canada. Canadian Association of Radiologists Journal, 64, 82-84. https://doi.org/10.1016/j.carj.2013.02.006

[3] Mayo, J.R. and Munk, P.L. (2010) Towards Clarity: What Does "Inappropriate Imaging” Really Mean? Canadian Association of Radiologists Journal, 61, 250-251. https://doi.org/10.1016/j.carj.2010.10.004

[4] Vanderby, S., Pena-Sanchez, J.N., Kalra, N. and Babyn, P. (2015) Finding the Truth in Medical Imaging: Painting the Picture of Appropriateness for Magnetic Resonance 
Imaging in Canada. Canadian Association of Radiologists Journal, 66, 323-331. https://doi.org/10.1016/j.carj.2015.05.002

[5] Perry, J.J. and Stiell, I.G. (2006) Impact of Clinical Decision Rules on Clinical Care of Traumatic Injuries to the Foot and Ankle, Knee, Cervical Spine, and Head. Injury, 37, 1157-1165. https://doi.org/10.1016/j.injury.2006.07.028

[6] Eagles, D., Stiell, I.G., Clement, C.M., Brehaut, J., Kelly, A.M., Mason, S., Perry, J.J., et al. (2008) International Survey of Emergency Physicians' Priorities for Clinical Decision Rules. Academic Emergency Medicine, 15, 177-182. https://doi.org/10.1111/j.1553-2712.2008.00035.x

[7] Butler, G.J. and Stolberg, H.O. (2004) The Use of Cross-Sectional Diagnostic Imaging in a Major Canadian Health Care Centre. Canadian Association of Radiologists Journal, 55, 174.

[8] You, J.J., Gladstone, J., Symons, S., Rotstein, D., Laupacis, A. and Bell, C.M. (2011) Patterns of Care and Outcomes after Computed Tomography Scans for Headache. The American Journal of Medicine, 124, 58-63. https://doi.org/10.1016/j.amjmed.2010.08.010

[9] Lammers, E.J., Adler-Milstein, J. and Kocher, K.E. (2014) Does Health Information Exchange Reduce Redundant Imaging? Evidence from Emergency Departments. Medical Care, 52, 227-234. https://doi.org/10.1097/MLR.0000000000000067

[10] Yaraghi, N. (2015) An Empirical Analysis of the Financial Benefits of Health Information Exchange in Emergency Departments. Journal of the American Medical Informatics Association, 22, 1169-1172. https://doi.org/10.1093/jamia/ocv068

[11] Sodickson, A., Opraseuth, J. and Ledbetter, S. (2011) Outside Imaging in Emergency Department Transfer Patients: CD Import Reduces Rates of Subsequent Imaging Utilization. Radiology, 260, 408-413. https://doi.org/10.1148/radiol.11101956

[12] American College of Radiology. ACR Appropriateness Criteria. https://acsearch.acr.org/list

[13] Canadian Association of Radiologist. 2012 CAR Diagnostic Imaging Referral Guidelines. https://car.ca/patient-care/referral-guidelines/

[14] Canadian Association of Radiologist. Radiology-Choosing Wisely Canada. https://choosingwiselycanada.org/radiology/

[15] Bowen, S., Johnson, K., Reed, M.H., Zhang, L. and Curry, L. (2011) The Effect of Incorporating Guidelines into a Computerized Order Entry System for Diagnostic Imaging. Journal of the American College of Radiology, 8, 251-258.

https://doi.org/10.1016/j.jacr.2010.11.020

[16] Hentel, K., Menard, A. and Khorasani, R. (2017) New CMS Clinical Decision Support Regulations: A Potential Opportunity with Major Challenges. Radiology, 283, 10-13. https://doi.org/10.1148/radiol.2017161560

[17] Gleen, C., Beck, M., Bruno, M., Mahraj, R., Caputo, G., DeFlitch, C. and Hollenbeak, C. (2016) Mandatory Assignment of Modified Wells Score Before CT Angiography for Pulmonary Embolism Fails to Improve Utilization or Percentage of Positive Cases. American Journal of Roentgenology, 207, 442-449. https://doi.org/10.2214/AJR.15.15394

[18] Protecting Access to Medicare Act of 2014, H.R. 4302, 113th Cong. (2014).

[19] The Protecting Access to Medicare Act (PAMA) [Internet]. National Decision Support Company, n.d. [cited 2017 September 1]. http://nationaldecisionsupport.com/pama/

[20] Slovis, B.H., Lowry, T., Delman, B.N., Beitia, A.O., Kuperman, G., Dimaggio, C. and Shapiro, J.S. (2016) Patient Crossover and Potentially Avoidable Repeat Computed 
Tomography Exams across a Health Information Exchange. Journal of the American Medical Informatics Association, 24, 30-38.

https://doi.org/10.1093/jamia/ocw035

[21] Jung, H., Vest, J.R., Unruh, M.A., Kern, L.M. and Kaushal, R. (2015) Use of Health Information Exchange and Repeat Imaging Costs. Journal of the American College of Radiology, 12, 1364-1370. https://doi.org/10.1016/j.jacr.2015.09.010

[22] DiRoberto, C., Lehto, C. and Baccei, S. (2016) Improving the Transcription of Patient Information From Image Requisitions to the Radiology Information System. Journal of the American College of Radiology, 13, 950-955.

https://doi.org/10.1016/j.jacr.2016.03.030 